\title{
The faecal abundance of short chain fatty acids is increased in men with a non-dipping blood pressure profile
}

Justine Huart, Arianna Cirillo, Annie Saint-Remy, Jean-Marie Krzesinski, Pascal de Tullio \& François Jouret

To cite this article: Justine Huart, Arianna Cirillo, Annie Saint-Remy, Jean-Marie Krzesinski, Pascal de Tullio \& François Jouret (2021): The faecal abundance of short chain fatty acids is increased in men with a non-dipping blood pressure profile, Acta Cardiologica, DOI:

10.1080/00015385.2021.1901020

To link to this article: https://doi.org/10.1080/00015385.2021.1901020

Published online: 31 Mar 2021.

Submit your article to this journal $\widetilde{ }$

View related articles

View Crossmark data $\nearrow$ 


\title{
The faecal abundance of short chain fatty acids is increased in men with a non-dipping blood pressure profile
}

\author{
Justine Huart ${ }^{a, b *}$, Arianna Cirilloc*, Annie Saint-Remy ${ }^{a}$, Jean-Marie Krzesinski ${ }^{a, b}$, Pascal de Tullioc $†$ and \\ François Jouret ${ }^{\mathrm{a}, \mathrm{b}}+$

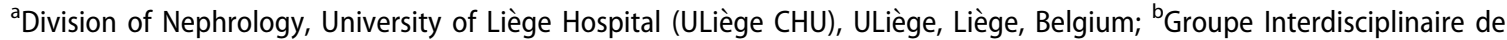 \\ Génoprotéomique Appliquée (GIGA), Cardiovascular Laboratory of Translational Research in Nephrology (LTRN), ULiège, Liège, \\ Belgium; 'Center for Interdisciplinary Research on Medicines (CIRM), Metabolomics group, ULiège, Liège, Belgium
}

\section{ABSTRACT}

Background and aims: Gut microbiota (GM) has been involved in the pathophysiology of hypertension (HT), notably via short chain fatty acids (SCFAs). Among the clinical manifestations of HT, the absence of a significant drop in night-time blood pressure (BP) (also known as the non-dipping BP profile) has been associated with poor renal and cardiovascular outcomes. The putative link between GM-derived metabolites and BP dipping status is still unknown.

Methods: Male volunteers $(n=44)$ were prospectively subjected to 24-hour ambulatory blood pressure monitoring, stool sample collection and a medical questionnaire. Metabolomics analyses of stool samples were conducted using Nuclear Magnetic Resonance (NMR).

Results: Higher amounts of acetate, butyrate and propionate were found in the stools of nondippers $(n=12)$ versus dippers $(n=26)(p=0.0252, p=0.0468$, and $p=0.0496$, respectively; $n=38$ in toto). NMR spectral data were not interpretable in 5 dippers and 1 non-dipper. A similar significant association was found when including only patients without anti-HT medications ( $p=0.0414, p=0.0108$, and $p=0.0602$, respectively; $n=21$ in toto). A not significant trend was observed when focussing only on HT patients without anti-HT medications ( $p=0.0556 ; n=14$ in toto).

Conclusion: Our pilot study highlights a putative link between GM-derived SCFAs and the BP dipping status, independently of the BP status itself or the anti-hypertensive medications.
ARTICLE HISTORY

Received 7 January 2021

Revised 19 February 2021

Accepted 2 March 2021

\section{KEYWORDS}

Hypertension; gut microbiota; dipping; short chain fatty acid; nondipping blood pressure profile; metabolomics

\section{Introduction}

Gut microbiota (GM) has been involved in the pathophysiology of hypertension (HT), possibly via its role in the production of short chain fatty acids (SCFAs) from diet carbohydrate fermentation [1]. We and others have recently reported on significant correlations between the $24 \mathrm{~h}$ mean blood pressure (BP) levels and the entire stool metabolome, as well as between BP levels and the faecal abundance of bacteria belonging to the Clostridial order. Higher amounts of the 3 main SCFAs (i.e. acetate, butyrate and propionate) have been also found in the stools of patients with HT compared to normotensive (NT) controls [2,3]. The absence of a significant drop in night-time BP (also known as the non-dipping BP profile) measured by 24-hour ambulatory BP monitoring (24h-ABPM) has been associated with poor renal and cardiovascular outcomes, independently of HT [4]. In our previous study, no significant difference in GM composition was observed between dippers versus non-dippers [2]. However, we did not compare the faecal metabolome of dippers versus non-dippers. So, the putative link between GMderived metabolites and BP dipping status remains unknown.

\section{Methods}

We investigated a cohort of male volunteers who were prospectively recruited and subjected to $24 \mathrm{~h}$ ABPM, stool sample collection and a medical questionnaire. A patient was categorised as non-dipper if the ratio between night and day systolic BP was $>0.9$. The patients were categorised in two groups, i.e. NT or HT, on the basis of the European Society of Hypertension criteria (https://www.eshonline.org). NT was defined by 
Table 1. Clinical characteristics of the cohort (dippers vs non-dippers).

\begin{tabular}{lccc}
\hline & Dippers & Non-dippers & $p$ Value \\
\hline $\mathrm{N}$ & 31 & 13 & \\
Age (years) & $50.6 \pm 9.9$ & $53 \pm 10.4$ & 0.95 \\
$\mathrm{BMI}\left(\mathrm{kg} / \mathrm{m}^{2}\right.$ ) & $26 \pm 3.4$ & $26.9 \pm 3.8$ & 0.39 \\
Smokers (\%, N) & $16.1 \%(5)$ & $7.7 \%(1)$ & 0.46 \\
Alcohol (glass/week) & $4.9 \pm 5$ & $4.1 \pm 4.3$ & 0.78 \\
Family HT (\%, N) & $42 \%(13)$ & $53.8 \%(7)$ & 0.47 \\
Diabetes (\%, N) & $3.2 \%(1)$ & 0 & $\mathrm{~ns}$ \\
CV history (\%, N) & $19.3 \%(6)$ & $30.7 \%(4)$ & 0.41 \\
GE history (\%, N) & $42 \%(13)$ & $46.1 \%(6)$ & 0.8 \\
24h Systolic BP (mmHg) & $131.6 \pm 12.1$ & $132.9 \pm 18.7$ & 0.96 \\
24h Diastolic BP (mmHg) & $81.5 \pm 9.5$ & $81.5 \pm 13.4$ & 0.74 \\
24h Mean BP (mmHg) & $97.6 \pm 10.2$ & $98.9 \pm 13.6$ & 0.89 \\
Night/Day Systolic BP ratio & $0.84 \pm 0.04$ & $0.96 \pm 0.04$ & $<0.0001$ \\
Anti-HT treatment (\%, N) & $38.8 \%(12)$ & $53.7 \%(7)$ & 0.35 \\
\hline BMl: body mass index; BP: blood pressure; CV: cardiovascular; GE: gastro- \\
enterological; HT: hypertension; ns: not significant. &
\end{tabular}

mean 24h BP levels $<130 / 80 \mathrm{mmHg}$ in untreated individuals with or without isolated nocturnal HT ( $\geq 120 \mathrm{mmHg}$ (systolic) and/or $\geq 70 \mathrm{mmHg}$ (diastolic)) but without isolated daytime HT $(\geq 135 \mathrm{mmHg}$ (systolic) and/or $\geq 85 \mathrm{mmHg}$ (diastolic)). HT was defined by mean $24 \mathrm{~h}$ BP levels $\geq 130 \mathrm{mmHg}$ (systolic) and/or $\geq 80 \mathrm{mmHg}$ (diastolic) or by isolated daytime HT ( $\geq 135 \mathrm{mmHg}$ (systolic) and/or $\geq 85 \mathrm{mmHg}$ (diastolic)) or in case of use of antihypertensive medications whatever the BP levels. A subgroup analysis was performed between the patients with versus without antihypertensive medications. Metabolomics analyses were conducted on stool supernatants using Nuclear Magnetic Resonance (NMR) and the CPMG relaxationediting sequence with presaturation, as previously detailed [2]. Faecal concentrations of acetate, butyrate and propionate were obtained by integrating the signals at $1.93 \mathrm{ppm}, 1.56 \mathrm{ppm}$ and $1.05 \mathrm{ppm}$, respectively. Univariate metabolomics data were subjected to a Mann-Whitney test. Mann-Whitney test and Chisquare test were used to compare continuous and categorical clinical variables, respectively.

\section{Results}

Our 44-case cohort included 13 non-dippers (29.6\%) and 31 dippers, with 35 HT (79.4\%) and 9 NT patients. Ten non-dippers (28.6\%) and 25 dippers were HT. Nineteen HT patients were under anti-hypertensive medications (43.1\%), including 7 non-dippers and 12 dippers. The mean age and body mass index (BMI) of the cohort were $50.8 \pm 9.5$ years and $26.3 \pm 3.5 \mathrm{~kg} / \mathrm{m}^{2}$, respectively. No significant difference in BP values, age, BMI, smoking habits, alcohol consumption, familial HT, personal history of diabetes, cardiovascular or gastro-intestinal disorders was observed between
Table 2. Blood pressure values between patients with antihypertensive medications (treated) and without anti-hypertensive medications (untreated).

\begin{tabular}{lrrc}
\hline & \multicolumn{1}{c}{ Treated } & \multicolumn{1}{c}{ Untreated } & $p$ Value \\
\hline $\mathrm{N}$ & \multicolumn{1}{c}{19} & \multicolumn{1}{c}{25} & \\
24h Systolic BP $(\mathrm{mmHg})$ & $134.5 \pm 15.8$ & $130.1 \pm 12.8$ & 0.37 \\
24h Diastolic BP $(\mathrm{mmHg})$ & $81.8 \pm 11.6$ & $81.3 \pm 10.1$ & 0.99 \\
24h Mean BP $(\mathrm{mmHg})$ & $99.5 \pm 11.8$ & $96.9 \pm 10.8$ & 0.44 \\
Daytime Systolic BP $(\mathrm{mmHg})$ & $140.3 \pm 16.1$ & $136 \pm 13.8$ & 0.43 \\
Daytime Diastolic BP $(\mathrm{mmHg})$ & $86.5 \pm 11.9$ & $86.8 \pm 11.1$ & 0.95 \\
Daytime Mean BP $(\mathrm{mmHg})$ & $104.3 \pm 12.2$ & $101.8 \pm 12.4$ & 0.51 \\
Night-time Systolic BP $(\mathrm{mmHg})$ & $123.4 \pm 17.6$ & $118.1 \pm 12.5$ & 0.37 \\
Night-time Diastolic BP $(\mathrm{mmHg})$ & $72.9 \pm 11.9$ & $70.8 \pm 10.5$ & 0.72 \\
Night-time Mean BP $(\mathrm{mmHg})$ & $90.3 \pm 13.1$ & $85 \pm 12.4$ & 0.21 \\
\hline BP. blood pressure. & & &
\end{tabular}

BP: blood pressure.

dippers and non-dippers (Table 1). The comparison of BP levels between patients with anti-hypertensive medications (i.e. 'treated') and those without any antihypertensive treatment (i.e. 'untreated') did not show any significant differences (Table 2). The relative quantification of faecal SCFAs showed higher amounts of acetate, butyrate and propionate in the stools of nondippers $(n=12)$ versus dippers $(n=26) \quad(p=0.0252$, $p=0.0468$, and $p=0.0496$, respectively; $n=38$ in toto) (Figure 1(A)). Multivariate statistical analyses by the means of PCA-X model of reduced and normalised spectral data lead to the exclusion of 5 dippers and 1 non-dipper because of uninterpretable data. The faecal amounts of acetate, butyrate and propionate were higher in non-dippers $(n=5)$ versus dippers $(n=16)$ in patients without anti-hypertensive medications ( $p=0.0414, p=0.0108$, and $p=0.0602$, respectively; $n=21$ in toto) (Figure 1(B)). When focussing only on HT patients without any anti-hypertensive medications, a not significant trend for higher amounts of the 3 main SCFAs was still found in the stools of nondippers $(n=2)$ versus dippers $(n=12) \quad(p=0.0556$; $n=14$ in toto).

\section{Discussion}

To the best of our knowledge, the BP dipping status has never been associated with GM composition or its derived metabolites. The non-dipping BP pattern reflects a disruption in the circadian BP rhythm $[4,5]$. Several molecular components of the circadian timing system controlled by the central and peripheral clocks have been involved in metabolic abnormalities leading to non-dipping BP profile [4]. This circadian misalignment between clocks is not well understood. However, it may implicate the GM $[6,7]$. Indeed, the relative abundance of $60 \%$ of the total gut bacteria and their metabolites undergo circadian variations, which may be disrupted in case of dysbiosis and/or circadian misalignment [6]. 


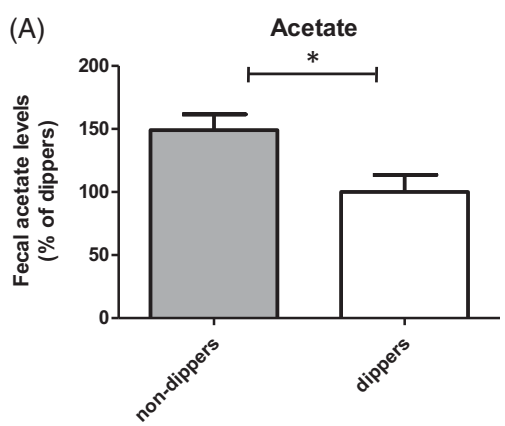

(B)

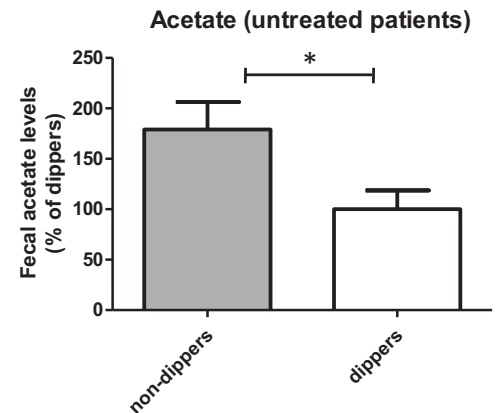

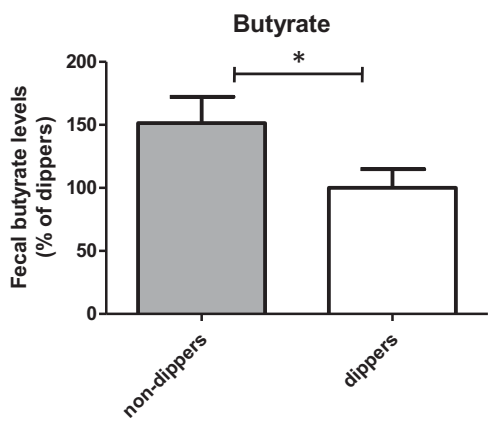

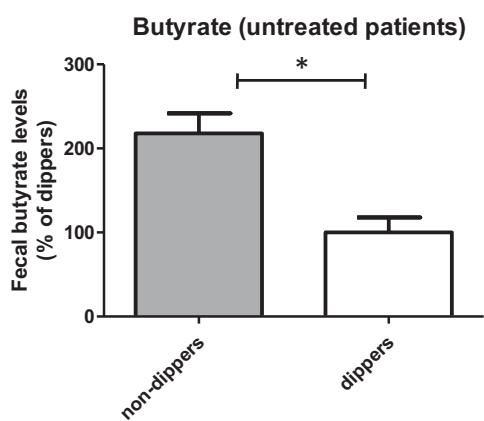

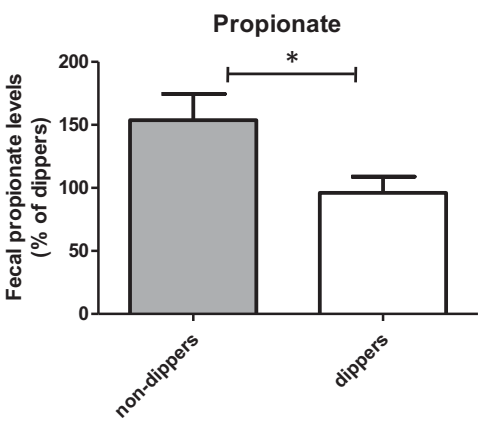

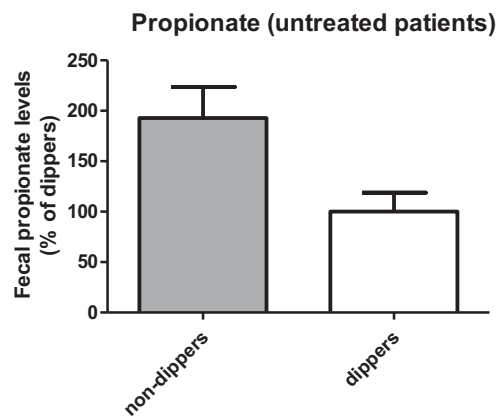

Figure 1. (A) Relative amounts of acetate, butyrate and propionate in the stools of non-dippers $(n=12)$ versus dippers $(n=26)$ in the whole cohort. $* p<0.05$. (B) Relative amounts of acetate, butyrate and propionate in the stools of non-dippers ( $\mathrm{n}=5$ ) versus dippers $(n=16)$ in the subgroup of patients without anti-hypertensive medications (untreated patients). ${ }^{*} p<0.05$.

Moreover, the integrity of $\mathrm{GM}$ and its production of metabolites, including the SCFAs, seem essential for the circadian rhythmicity of gene expression in several organs $[8,9]$. In both mice and humans, Thaiss et al. showed that jetlag-induced dysbiosis promotes the development of obesity and glucose intolerance, which are transferrable to germ-free mice through faecal transplantation [10]. On the basis of these observations, one may speculate that disruption in the circadian BP rhythm (i.e. the non-dipping BP profile) may be associated with a disrupted rhythmicity of GM-mediated metabolite secretion, including SCFAs. None of our patients presented metabolic dysfunctions or confounding factors, when comparing dippers versus non-dippers. Although confirmatory data in larger cohorts are obviously needed, our observation of a putative link between GM and stool metabolome and BP dipping may unravel innovative pathophysiological investigations in the field.

\section{Acknowledgements}

We sincerely thank all patients who joined the study. We thank Laurence Poma (LTRN lab) for her technical assistance and commitment to this project.

\section{Disclosure statement}

All the authors declare that they have no conflict of interest involving the work under consideration for publication.

\section{Funding}

Grant Support: Fonds Léon Frédéricq. JH and FJ are Fellows of the Fonds National de la Recherche Scientifique (FNRS), Belgium. PdT is Research Director of the Fonds National de la Recherche Scientifique (FNRS), Belgium. AC is supported by Belgian grants of the "Fonds pour la recherche dans I'industrie et l'agriculture » (FRIA).

\section{References}

[1] Meijers B, Jouret F, Evenepoel P. Linking gut microbiota to cardiovascular disease and hypertension: lessons from chronic kidney disease. Pharmacol Res. 2018;133:101-107.

[2] Huart J, Leenders J, Taminiau B, et al. Gut microbiota and fecal levels of short-chain fatty acids differ upon 24-hour blood pressure levels in men. Hypertension. 2019;74(4):1005-1013.

[3] Verhaar BJH, Collard D, Prodan A, et al. Associations between gut microbiota, faecal short-chain fatty acids, and blood pressure across ethnic groups: the HELIUS study. Eur Heart J. 2020;41(44):4259-4267.

[4] Rahman A, Hasan AU, Nishiyama A, et al. Altered circadian timing system-mediated non-dipping pattern of blood pressure and associated cardiovascular disorders in metabolic and kidney diseases. IJMS. 2018; 19(2):400.

[5] Agarwal R. Regulation of circadian blood pressure: from mice to astronauts. Curr Opin Nephrol Hypertens. 2010;19:51-58.

[6] Paschos GK, FitzGerald GA. Circadian clocks and metabolism: implications for microbiome and aging. Trends Genet. 2017;33(10):760-769. 
[7] Voigt RM, Forsyth CB, Green SJ, et al. Circadian disorganization alters intestinal microbiota. PLoS One. 2014;9(5):e97500.

[8] Leone V, Gibbons SM, Martinez K, et al. Effects of diurnal variation of gut microbes and high-fat feeding on host circadian clock function and metabolism. Cell Host Microbe. 2015;17(5):681-689.
[9] Mukherji A, Kobiita A, Ye T, et al. Homeostasis in intestinal epithelium is orchestrated by the circadian clock and microbiota cues transduced by TLRs. Cell. 2013;153(4):812-827.

[10] Thaiss CA, Zeevi D, Levy $M$, et al. Transkingdom control of microbiota diurnal oscillations promotes metabolic homeostasis. Cell. 2014;159(3):514-529. 